\title{
Ovarian endometriomas: the role of aspiration (sclerosing) therapy
}

\begin{abstract}
The article discusses disputable problems of aspiration therapy of ovarian endometriomas. A comparatively high (43.2\%) incidence of relapses of endometriomsa within a 12-month follow-up after sclerosing therapy has been noted. At the same time, pregnancy occurred in $37.3 \%$ of women during this period, later ending with childbirth in $100 \%$ of cases. Aspiration therapy of ovarian endometriomsa presupposes a careful selection of patients for such intervention. Its favourable outcomes in many respects depend on hormone therapy compliance.
\end{abstract}

Keywords: endometriomas, aspiration (sclerosing) therapy, ovarian reserve
Volume 3 Issue 3 - 2018

\author{
Davydov Al, Shahlamova MN \\ Professor of Medicine at the Chair of Obstetrics, Gynaecology \\ and Perinatology, I.M. Sechenov First Moscow State Medical \\ University, Russia
}

\begin{abstract}
Correspondence: Davydov Aleksandr ll'gizirovich, Professor of Medicine at the Chair of Obstetrics, Gynaecology and Perinatology, I.M. Sechenov First Moscow State Medical University, Russia, Email al-davydov@mail.ru
\end{abstract}

Received: February 16, 2018 | Published: June 06, 2018

\section{Introduction}

Ovarian endometriosis is a particular form of endometriosis, which is characterized by specific clinical, pathophysiological and histological characteristics:

i. Ovarian endometriosis is the most frequent localization of endometriosis lesions;

ii. Ovarian endometriosis adversely affects the folliculogenesis in general and the function of yellow body in particular;

iii. Ovarian endometriosis inhibits ovarian reserve, destroying the functional tissue of the ovaries;

iv. it is believed that originally it is ovarian endometriosis, which causes the development of severe complication - deep infiltrative endometriosis;

v. Finally, ovarian endometriosis, in contrast to other localizations, presents the greatest potential threat of malignization.

Features of the histogenesis of the ovarian endometriomas (capacity of infiltration into surrounding tissues) lead to the decrease of the amount of functioning ovarian tissue, regardless of the size of the formation. Another concept of ovarian dysfunction in cases of endometriosis is that endometriomas contain a huge amount of free iron, which forms toxic superoxide by reaction with hydrogen peroxide, leading to oxidative stress and thus to disruption in the functioning of the cell. The impact of reactive oxygen species on the activity of anti-inflammatory cytokines, adhesion molecules, growth factors and angiogenic factors leads to inhibition of cell growth in the ovary. ${ }^{1}$ Therefore the presence of endometrioma can already be considered as a damage factor of ovarian reserve.

One more important problem of endometriomas is oncological alertness. Today the risk of malignization of endometriosis is not the reason for the debate. However, in the discussion of this problem is a "slipping away" important clarification: the ability to malignization usually has ovarian endometriosis. For ovarian endometriosis is typical cellular irregularities, which are usually regarded as the phase transition of benign to malignant process. ${ }^{2}$
It seems that the presence of such features has to establish absolute indications for surgical treatment of patients with ovarian endometriosis. However, the question of tactics of treatment is discussed up to the present time. There is a point of view, according to which ovarian endometriomas with a diameter up to $3 \mathrm{~cm}$ may conduct drug therapy. The main argument of this position is the sharply negative impact of surgery on ovarian reserve and ovaries. It is impossible not to admit that this fact has a high level of confidence.

Mechanisms of loss of follicular reserve after surgical treatment of patients with ovarian endometriomas are different. However, the main reason is closely associated with morphogenesis of endometriomas. Endometrioma, in contrast to other tumors, is characterized by the presence of subepithelial dense adhesions between the cyst capsule and the hilus - the place of passage of major blood and lymphatic vessels, supplying the ovary. Therefore, cystectomy in cases of endometriotic cysts is a big trauma to the ovary, and the need for ligation (coagulation) of the ovary in the area of its portal causes additional disturbances in the ovarian blood supply.

So, on the one hand, passive observation or medical therapy of endometriomas is associated with the high risk of various complications (the decrease of ovarian function, an expanisve growth, the threat of malignization); on the other hand, classic cystectomy is fraught with potential loss of ovarian reserve. Therefore, to solve this problem a compromise is required. Today, the role of the "Golden mean" of the treatment of patients with ovarian endometriomas performs aspiration (sclerosing) therapy.

In the literature of recent years, there are a relatively large number of publications on this subject. The authors of the present work carried out the first such intervention in 1999 in a female patient, 32 years old, with single ovary, which was diagnosed with a recurrence of endometriotic cysts after previous cystectomy. ${ }^{3}$ The first experiment was successful: after 2 years of infertility, pregnancy came and ended with a normal delivery.

From that period there were 118 such interventions, a retrospective analysis of which helped to justify clear indications for sclerosing therapy of ovarian endometriomas: 
i. Reproductive age (especially its beginning), with no pregnancies in anamnesis;

ii. Recurrence of endometriotic cyst of the single ovary;

iii. Previous multiple surgeries on a single ovary;

iv. High risk of complications of abdominal surgery;

v. Planning of IVF;

vi. Rapid recurrence of histologically verified endometrioma.

Of course, in each particular case should prevail individual approach to the choice of treatment. It should be emphasized that the aspiration therapy for endometriomas implies the use of postoperative hormonal therapy. Appointment postoperative exogenous hormones (antihormonal) pursues the following objectives:

a. To add the sclerosing effect of ethanol;

b. To suppress the peak emission of hormones of the anterior pituitary, which contributes to cause a relapse of the disease.

In the intervening period we have completed the analysis of 118 cases of the aspiration therapy of patients with endometrioid ovarian cysts. The frequency of relapses during the first 12 months of observation was $43.2 \%$. During the same period, the pregnancy occurred in approximately $37.3 \%$ of women, which subsequently resulted in delivery of $100 \%$. Some patients $(19,5 \%)$, for various reasons, did not plan pregnancy (Figure 1).

How to treat a recurrence rate of aspiration therapy? On the one hand, it is high enough; on the other hand, it is not much higher than after cystectomy. Furthermore, this method provides careful treatment of the ovarian reserve in conjunction with chemical degradation of the capsule of the cyst. Considering the relatively high recurrence rate of endometriomas after aspiration therapy the following aspects are required: first, careful selection of patients for such an intervention; secondly, the preparatory interviews with the patient, in which a detailed way all the advantages and disadvantages of lean operation are described.

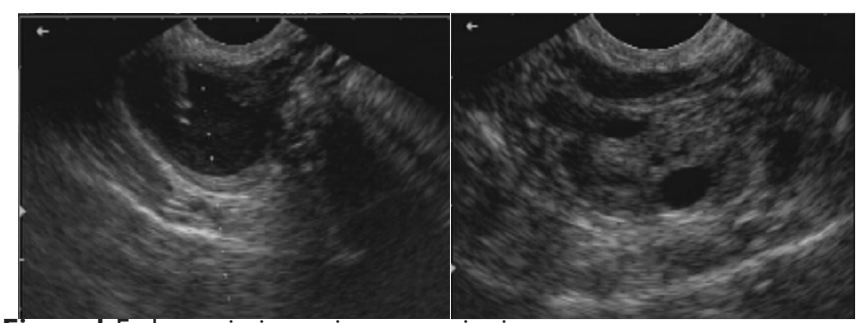

Figure I Endometriotic ovarian cyst aspiration:

(A) Needle introduced,

(B) The image of the ovary after sclerosing therapy.

\section{Conclusion}

So, sclerosing therapy of endometriomas has its clearly localized niche in the complex treatment of women with ovarian endometriosis. In some situations, this method can have the status of "finished" therapeutic action, in the series-it is a preparatory stage for cystectomy. Favourable outcomes of the aspiration therapy highly depends on compliance of hormone treatment.

\section{Acknowledgements}

None.

\section{Conflict of interest}

The author declares no conflict of interest.

\section{References}

1. Goud PT, Goud AP, Joshi N, et al. Dynamics of nitric oxide, altered follicular microenvironment, and oocyte quality in women with endometriosis. Fertil Steril. 2014;102(1):151-159.

2. Matias GX, Stewart CJR. Endometriosis-associated ovarian neoplasia. Pathology. 2018;(2):190-204.

3. Davydov AI. Ultrasonic mini-surgery for the treatment of endometriotic ovarian cysts in patients with multiple laparotomies in anamnesis. New technologies in gynecology. Moscow; 2000. 